\title{
Comparison of Naturally Conceived and IVF-DZ Twins in the Netherlands Twin Registry: A Developmental Study
}

\author{
Catharina E. M. van Beijsterveldt, Meike Bartels, and Dorret I. Boomsma \\ Department of Biological Psychology, VU University, 1081 BT Amsterdam, The Netherlands \\ Correspondence should be addressed to Catharina E. M. van Beijsterveldt, t.van.beijsterveldt@vu.nl
}

Received 14 July 2011; Accepted 11 August 2011

Academic Editor: Ariel Zosmer

Copyright ( 2011 Catharina E. M. van Beijsterveldt et al. This is an open access article distributed under the Creative Commons Attribution License, which permits unrestricted use, distribution, and reproduction in any medium, provided the original work is properly cited.

\begin{abstract}
In a large set of twin pairs, we compared twins born after IVF to naturally conceived twins with respect to birth characteristics, growth, attainment of motor milestones, and emotional and behavioral problems. Twin families were registered with the Netherlands Twin Register. We included 1534 dizygotic (DZ) twins born after IVF, 5315 naturally conceived (NC) DZ twins, and 1504 control NC DZ twins who were matched to the IVF twins based on maternal age, maternal educational level, smoking during pregnancy, gestational age, and offspring sex. Data were obtained by longitudinal surveys sent to fathers, mothers, and teachers at ages $1,2,3,7,10$, and 12 years. Results showed no differences in growth, in attainment of motor milestones, and in behavioral development between IVF and matched NC twins. It can be concluded that for nearly all aspects, development in IVF and NC children is similar.
\end{abstract}

\section{Introduction}

In the Netherlands, the number of children born after assisted reproductive technologies (ART) including in vitro fertilization (IVF) and intracytoplasmic sperm injection (ICSI) is rapidly increasing. In 1996, 1 in 77 newborns was born after IVF or ICSI treatment and in 2005, this had increased to 1 in $43[1,2]$. The first IVF treatment in the Netherlands was in 1983, and ICSI was introduced in 1994 [3]. Nowadays, ICSI is often used simultaneously with IVF treatment [2]. The introduction of ART was accompanied by an increase in the number of multiple births. Between 1987 and 1994, the percentage of multiple births after IVF/ICSI treatments fluctuated around $25 \%$ [4]. This percentage dropped to $18.5 \%$ in 2005 but a significant proportion of ART treatments still results in a twin pregnancy. There is no doubt that twin pregnancies have a higher risk of complications compared to singleton pregnancies. However, only a few studies have compared the long-term development between twins following IVF/ICSI treatment and twins following natural conception (NC) [5]. The main aim of the present study was to investigate the short- as well as the long-term development of twins born after IVF/ICSI treatment and after NC.
Previous research on perinatal and obstetric outcomes of twin pregnancies after assisted reproduction has produced mixed results. The comparison of the outcomes of IVF/ICSI twin pregnancies and NC twin pregnancies is complex because IVF/ICSI mothers are older than mothers of NC twins, are more often primiparous, and have a history of infertility problems, all factors that may negatively influence perinatal and obstetric outcomes [6]. In addition, studies that included both $\mathrm{MZ}$ and $\mathrm{DZ}$ twins as controls may be biased as there are more adverse effects in MZ pregnancies [7]. Even when comparisons are restricted to DZ twins only, studies have shown large differences in outcomes. Some studies reported a higher rate of preterm birth and lower birth weight in IVF/ICSI twins [8-10], whereas others reported no differences in perinatal outcomes between the two groups [1113]. The question thus remains whether the adverse perinatal outcomes are due to maternal characteristics or due to the IVF procedure itself. A recent meta-analysis of perinatal risks in twins [14], which selected studies that matched or controlled for maternal age and often other factors, showed that IVF twins had an increased risk of preterm birth and low birth weight compared to NC twins. 
Journal of Pregnancy

In recent years, a growing number of studies investigated the longer-term development in growth, health, and psychosocial development of IVF/ICSI children $[5,15,16]$. The developmental trajectories in IVF/ICSI children could be different because of the IVF procedure itself, as consequence of the infertility problems or as an effect of problems in the perinatal period, such as lower birth weight and shorter gestational age. There is also evidence that parents of IVF children and NC children differ with respect to parental attitudes, parental concerns, and educational styles [1720].

Most research on IVF-related outcomes is done in singletons, and data on development of IVF twins are limited $[5,21]$. In the first 3 years of life, lower weight and height for IVF singletons compared to controls have been reported [22, 23 ], with the most pronounced differences during the first 6 months. However, studies comparing IVF and NC twins found no growth differences in the first years of life $[23,24]$. For growth between ages 5 and 18, no differences in weight and height were seen between IVF singletons and NC controls born to subfertile parents $[25,26]$, although Ceelen et al. [25] found evidence that IVF children had more peripheral body mass and fat as compared to controls [25]. For motor development, there were no differences between IVF/ICSI children and NC children during childhood [23, 27-30]. Studies comparing behavioral and emotional problems between IVF and NC children showed mixed results. Up to age 9, IVF singletons showed normal behavior and socioemotional functioning [17, 31-33]. Parents of IVF adolescents even reported fewer externalizing problems $[31,34]$. Parents and teachers of IVF singletons reported more withdrawn/depressed behavior than the parents of NC singletons [34], but when these children reported on their own behaviors [35], no differences were observed in behavioral functioning between the IVF and the control group [35]. In twins, parental ratings of externalizing and internalizing problem behaviors of 5-year olds were similar in IVF and NC twins. Teacher ratings of the twins' behavior did not differ between IVF and NC twins [36]. Taken together, the current data suggest that IVF singletons and twins show normal psychosocial development during childhood.

Up until now the short- and long-term development of IVF/ICSI children has mainly been studied in singletons. Because a significant proportion of IVF pregnancies results in a twin pregnancy, it is important to examine whether there are differences in development between twins after fertility treatment and NC twins. Comparing IVF twins to control samples of singletons may introduce bias as twins are at higher risk than singletons for low birth weight, low gestational age, and developmental delays. In this study we compare the development of IVF twins to carefully matched control twins. We look at perinatal outcomes, growth, motor development, and behavior problems during childhood. Because the proportion of MZ twins is low following IVF/ ICSI conception, only DZ twins were included. The IVF and the NC DZ twins were matched on birth cohort, maternal age and educational level, smoking behavior during pregnancy, and gestational age of the twins.

\section{Methods}

2.1. Participants. The data on mode of conception and development measures in twins come from a longitudinal study designed to examine the genetic and environmental influences on the development of behavioral and emotional problems in twins from birth onwards. The twin families are volunteer members of the Netherlands Twin Register (NTR) maintained by the Department of Biological Psychology at VU University in Amsterdam [37-39]. The NTR recruits families with twins a few months after birth. Depending on birth cohort, between 25 and $40 \%$ of all multiple births in The Netherlands are registered by the NTR. For the present study, data obtained at ages $1,2,3,7,10$, and 12 years were included for twins born between 1990 and 2000.

Data on mode of conception, mode of delivery, age at birth, gestational age, birth weight, birth order, sex of twins, and smoking behavior of both parents during pregnancy come from survey- 1 which is collected after parents register their twins (age $<1$ years). Information on maternal educational level was obtained at age 3 of the twins (survey-3), and if missing, educational level was supplemented with information obtained at ages 7 (survey-7) or 10 (survey-10).

For 11708 twin pairs complete data on the variables used for matching were available (i.e., mode of conception, gestational age, age of mother at birth, smoking behavior during pregnancy, zygosity, and maternal educational level). There were 9001 twin pairs who were born following natural conception, 1606 pairs born following IVF/ICSI (at least 288 pairs after ICSI), and 1101 pairs born after ovulation induction. For the analyses we excluded twin pairs conceived by ovulation induction and all MZ pairs $(N=72$ for the IVF/ICSI group; $N=3686$ for the NC group). Information on zygosity of 808 same-sex twin pairs was based on blood group/DNA group polymorphisms. For the remaining samesex twin pairs $(N=6998)$, zygosity was assessed using items about physical similarity and frequency of confusion of the twins by family and strangers [40], collected in surveys at 3 , $5,7,10$, and 12 years.

In the analyses, there were $1534 \mathrm{DZ} \mathrm{IVF/ICSI} \mathrm{twin} \mathrm{pairs}$ (1606 minus $72 \mathrm{MZ}$ twin pairs); these are referred to as IVF twins throughout the paper. From a total of $5315 \mathrm{NC}$ DZ twin pairs, a control group of NC DZ twin pairs was formed by matching for birth cohort, gestational age, age of mother at birth, smoking behavior during pregnancy, zygosity, and maternal educational level $(N=1504)$.

\subsection{Measures}

2.2.1. Mode of Conception. Survey-1 included one question about the use of hormonal preparations. Possible answers were (1) no hormonal preparations, (2) oral contraceptives before getting pregnant, (3) ovulation induction, and (4) ovulation induction in combination with IVF. Endorsement of more than one answer was possible. In 2005, a 2-page survey with questions about familial twinning, fertility, and twin pregnancy was sent to all mothers of twins who were registered with the NTR [41]. This survey included one item on mode of conception, with the following answers: 
(1) naturally conceived, (2) IVF, (3) ICSI, (4) IUI, (5) ovulation induction, or (6) other, with additional space for comments [42]. For mothers who only returned survey-1, we formed the groups of naturally conceived twins, IVF twins, and twins born after ovulation induction. For about $70 \%$ of the twin pairs, the mother returned both surveys. For these twin pairs we could make an additional distinction between IVF and ICSI twin pairs.

2.2.2. Motor Milestones. In survey-2, mailed out when the twins were 2 years old, the mother was asked to report the age at which certain motor milestones were reached (turning over from back to belly (turning), sitting without support (sitting), crawling on hands and knees (crawling), standing without support (standing) and walking without support (walking)) [43, 44]. With survey-1, mothers received a memory aid to track the motor milestones. For 476 children (from 238 twin pairs), the mailed survey data were compared with monthly telephone interview data collected from the mothers on the time which motor milestones were achieved. With exception of "standing", no differences in times were found between the two assessment methods [43].

2.2.3. Behavior Problems Rated by the Parents and Teachers. At age 3, externalizing and internalizing behavior problems were assessed using the $\mathrm{CBCL} / 2-3$ [45]. Both parents were asked to rate the behavior of the children for the preceding 6 months on a 3-point scale. The CBCL includes two broad categories of problem behaviors: externalizing behaviors (including the syndromes: aggressive behavior, oppositional and overactive problems) and internalizing behaviors (including the syndromes: anxious and withdrawn/depressed). The syndromes are constructed for the Dutch population [46] and comparable with the syndrome scales as developed by Achenbach [45].

Behavior problems were measured at ages of 7,10 , and 12 years using the CBCL/4-18 [47]. The scales overlap with the $\mathrm{CBCL} / 2-3$ to a large extent. Externalizing behaviors include the syndrome scales: rule breaking and aggressive behavior and internalizing behavior includes withdrawn, somatic complaints, and anxious/depressed behavior. In addition, data from the Attention Problems scale were analyzed.

After consent was obtained from parents, teachers of twins were asked to fill in a questionnaire about the twins' behavioral problems. Teachers were required to have known the children for at least 3 months. At the ages of 7, 10, and 12 years, teachers rated behavioral problems using the Teacher's Report Form (TRF [48]). The TRF scales are comparable with the scales of the CBCL4-18, although item content can differ slightly.

2.2.4. Educational Level. Maternal education level was measured on a 13-point scale, ranging from primary education to postdoctoral education. Educational level was classified into three categories (low, middle, and high).

2.2.5. Growth. Mothers of twin pairs were asked to report offspring height and weight in the surveys at ages 1, 2, 3, 7,
10, and 12 years. Data were converted to Standard Deviations Scores (SDS) by comparison of weight and height to the general population using the software package growth analyzer 3.5 containing the Dutch reference growth charts for the general population from 1997 [49, 50]. The SDS scores indicate by how many standard deviations the relevant measurement differs from the mean of the Dutch reference growth charts.

2.3. Statistical Analyses. The data were analyzed using SPSS version 17.0 (statistical packages for social sciences). As first step we compared the maternal and demographic characteristics of twins in the IVF group versus all DZ $\mathrm{NC}$ twins. In a second step, the IVF group was compared to a group of matched NC twins. Matching of IVF and NC pairs was done by using the "duplicate case" option in SPSS. Differences in proportions for parental and birth characteristics between IVF and NC twins were tested using chi-squared tests. For the continuous dependent variables, ANOVA was used to compare the means for maternal and twin pair characteristics. The mixed models procedure and generalized estimating equations (GEE; SPSS [51]) were used for the comparisons of the characteristics of the individual children between IVF and matched NC groups. In twin data observations are not statistically independent as there are two children from the same family. Using mixed models, and GEE it is possible to adjust for this dependency in the data of twin pairs. To evaluate the importance of significant findings, the effect size (Cohen's $d$ ) was computed. This was done by computing the difference between estimated means divided by the square root of the standard deviations of the 2 groups. An effect size of 0.20 is considered small, of 0.50 moderate, and 0.80 large. To correct for multiple testing and to determine the significance of the results, Bonferroni correction was applied by dividing the significance level by the number of independent traits in each developmental domain.

\section{Results}

3.1. Parental, Birth, Child Characteristics. First, parental characteristics were compared between the IVF twins and the unmatched NC twins. Results are given in Table 1. In the IVF group, both mothers and fathers were older at the birth of the twin pair compared to the parents of unmatched NC twins. Mothers of unmatched NC twins smoked more often during pregnancy than the mothers of IVF twins. No differences were found in educational level between the two groups of mothers. This reflects the fact that in The Netherlands IVF treatment is paid for by health insurance, which is obligatory with private health insurance companies, and IVF is equally accessible to parents from different socioeconomic backgrounds. Gestational age was shorter and more preterm births were observed in the IVF group compared to the unmatched NC group. In addition, mothers of unmatched NC twins were taller and weighed more than mothers of IVF twins. No differences were found in weight gain during pregnancy between the two groups. 
TABLe 1: Parental, birth, and child characteristics of IVF/ICSI DZ twin pairs, naturally conceived (NC) DZ twin pairs, and a group of DZ twin pairs matched for birth cohort, educational level, maternal age, gestational age, and smoking behavior during pregnancy (matched NC pairs).

\begin{tabular}{|c|c|c|c|c|c|}
\hline & $\begin{array}{c}\text { NC pairs } \\
N=5315\end{array}$ & $\begin{array}{c}\text { IVF/ICSI pairs } \\
N=1534\end{array}$ & $P^{*}$ & $\begin{array}{l}\text { Matched NC pairs } \\
\qquad N=1504\end{array}$ & $P^{* *}$ \\
\hline Age mother $(y)($ mean \pm SD $)$ & $30.99(3.78)$ & $33.25(3.44)$ & $<0.001$ & $33.08(3.68)$ & 0.185 \\
\hline Age father $(y)($ mean $\pm S D)$ & $33.19(2.44)$ & $36.03(4.68)$ & $<0.001$ & $35.01(4.69)$ & 0.001 \\
\hline Smoked during pregnancy (\% yes) & $1344(25.3 \%)$ & $264(17.2 \%)$ & $<0.001$ & $283(18.8 \%)$ & 0.249 \\
\hline \multicolumn{6}{|l|}{ Educational level } \\
\hline$\%$ Low & $1999(37.6 \%)$ & $553(36.0 \%)$ & 0.515 & $544(36.2 \%)$ & 0.842 \\
\hline$\%$ Middle & $2184(41.1 \%)$ & $651(42.4 \%)$ & & $649(43.2 \%)$ & \\
\hline \% High & $1132(21.2 \%)$ & $330(21.5 \%)$ & & $311(20.7 \%)$ & \\
\hline Gestational age (weeks) (mean \pm SD) & $36.84(2.43)$ & $36.40(2.54)$ & $<0.001$ & $36.50(2.43)$ & 0.241 \\
\hline$\%>32$ and $<37$ weeks & $1539(29.0 \%)$ & $561(36.6 \%)$ & $<0.001$ & $551(36.6 \%)$ & 0.847 \\
\hline$\% \leq 32$ weeks & $326(6.1 \%)$ & $109(7.1 \%)$ & & $99(6.6 \%)$ & \\
\hline Child sex (\% boys) & $5464(51.4 \%)$ & $1586(51.7 \%)$ & 0.775 & $1566(52.1 \%)$ & 0.775 \\
\hline Older sibs $(\%)$ & $2199(57.2 \%)$ & $232(21.5 \%)$ & $<0.001$ & $691(63.3 \%)$ & $<0.001$ \\
\hline Height mother $(\mathrm{cm})($ mean $\pm S D)$ & $170.39(6.42)$ & $169.91(6.67)$ & 0.012 & $170.49(6.45)$ & 0.017 \\
\hline Weight before pregnancy $(\mathrm{kg})($ mean $\pm \mathrm{SD})$ & $69.13(11.77)$ & $68.52(11.69)$ & 0.077 & $69.40(11.95)$ & 0.042 \\
\hline Weight gain during pregnancy $(\mathrm{kg})($ mean $\pm \mathrm{SD})$ & $16.41(6.23)$ & $16.35(6.54)$ & 0.782 & $16.24(6.04)$ & 0.630 \\
\hline
\end{tabular}

Note: ${ }^{*} P$ value based on tests comparing IVF and unmatched NC twin pairs ${ }^{* *} P$ value based on tests comparing IVF and matched NC twin pairs.

To investigate the possible risks of IVF on health, growth, motor development, and problem behaviors we obtained a control group of 1504 twin pairs who were matched on maternal age, gestational age, educational level, zygosity, and smoking behavior. Comparisons of the IVF pairs with the NC pairs matched on maternal and birth characteristics are given in Table 1. After matching, there is still a large difference between the IVF and matched NC group in the number of older sibs. In contrast to the matched NC group, for the vast majority of IVF mothers the twin birth was the first birth.

\subsection{Birth Weight, Mode of Delivery, and Hospital Admission.} Both first- and second-born IVF twins had lower birth weights and lengths than matched NC twins, but the effect was augmented in second-born twins. However, the proportion of children with a birth weight lower than 1500 grams is not different between second-born IVF and matched NC controls. In addition, birth weight discordance was found to be larger in the IVF twins than in matched NC twins. When the analyses were restricted to primiparous mothers, the differences in birth length and weight and birth weight discordance disappeared, with the exception of birth weight of the first born twin $(P=0.023)$. As shown in Table 2, IVF twins were more often delivered by caesarean section compared to control twins. The difference in frequency of caesarean sections between the 2 groups remained when limiting the analyses to primiparous mothers. In the IVF group the rate was increased to $40.6 \%$ and in the control group to $33.8 \%$.

Admission rate to an incubator was the same for IVF and matched NC twins, but the number of days in an incubator was higher in the IVF group compared to the matched NC group. In addition, the proportion of twins that remained in an incubator for longer than 1 month was larger in the IVF group compared to the matched NC group. After controlling for parity, the difference in time in incubator was no longer statistically significant. At age 3, the proportion of children with a hospital admission was similar in IVF and matched NC twins.

3.3. Motor Milestones and Growth. We found significant differences for the ages at which three motor milestones were attained, with IVF twins doing better than matched NC twins (see Table 3). The effect sizes were $0.11,0.12$, and 0.08 for sitting, standing, and walking, respectively.

The SDS's for height and weight across ages 1 to 12 years are given in Table 4. At age 1, both IVF and matched NC twins were $0.5 \mathrm{SD}$ smaller and weighted less than children of the same age in the Dutch population. Until age 7, twins remained smaller than children in the Dutch population. After the age 7, the twins were similar in height. For weight, the pictures differ to that for height. Until the age of 12, the twins remained smaller than their peers in the Dutch population. These trends were the same for the IVF group and the control group (at a significance level of $0.008(0.05 / 6)$ ). Thus, growth patterns did not seem to differ between IVF and matched NC twins.

3.4. Behavioral Problems. The results for behavioral problems are presented in Table 5. Univariate analyses did not reveal any differences between the IVF and the matched NC twins. At all ages and for all raters, the IVF and the matched NC twins had the same scores for internalizing and externalizing behavior, and attention problems. At ages 3 and 7, IVF children tended to have slightly higher internalizing scores as rated by their mother and by their teachers. However, 
TABLE 2: Mode of delivery, birth weight and length, incubator, and hospital admission for IVF/ICSI twins and matched NC twins.

\begin{tabular}{|c|c|c|c|}
\hline & IVF/ICS twins & Matched NC twins & $P^{*}$ \\
\hline $\begin{array}{l}\text { Delivery (\% } \\
\text { caesarean-section) }\end{array}$ & $1085(36.1 \%)$ & $804(27.2 \%)$ & $<0.001$ \\
\hline \multicolumn{4}{|l|}{$\begin{array}{l}\text { Birth weight }(\mathrm{g}) \\
(\text { mean } \pm \mathrm{SD})\end{array}$} \\
\hline First born & $2494(557)$ & $2560(546)$ & 0.001 \\
\hline Second born & $2405(563)$ & $2507(542)$ & $<.001$ \\
\hline \multicolumn{4}{|l|}{$\begin{array}{l}\text { Birth weight } \\
(\%<1500 \mathrm{gr})\end{array}$} \\
\hline First born & $69(4.5 \%)$ & $44(3.0 \%)$ & 0.024 \\
\hline Second born & $90(5.9 \%)$ & $66(4.5 \%)$ & 0.074 \\
\hline $\begin{array}{l}\text { Birth weight } \\
\text { discordance } \\
(\text { mean } \pm S D)\end{array}$ & $13.01(10.40)$ & $12.05(9.46)$ & 0.009 \\
\hline $\begin{array}{l}\% \text { with } \geq 20 \% \\
\text { discordancy }\end{array}$ & $338(22.3 \%)$ & $276(18.8 \%)$ & 0.016 \\
\hline \multicolumn{4}{|l|}{$\begin{array}{l}\text { Birth length } \\
(\text { mean } \pm \mathrm{SD})\end{array}$} \\
\hline First born & $46.47(3.73)$ & $46.86(3.58)$ & 0.015 \\
\hline Second born & $46.25(3.83)$ & $46.76(3.51)$ & 0.002 \\
\hline Incubator (\% yes) & $1566(51.6 \%)$ & $1454(48.9 \%)$ & 0.039 \\
\hline $\begin{array}{l}\text { Incubator (days) } \\
(\text { means } \pm \mathrm{SD})\end{array}$ & $11.77(15.44)$ & $10.16(15.36)$ & 0.044 \\
\hline $\begin{array}{l}\text { Incubator: more than } \\
1 \text { month ( } \% \text { yes })\end{array}$ & $171(11.8 \%)$ & $109(8 \%)$ & 0.014 \\
\hline $\begin{array}{l}\text { Hospital admission at } \\
\text { age } 3 \text { (\% yes) }\end{array}$ & $422(15.2 \%)$ & $365(13.2)$ & 0.055 \\
\hline
\end{tabular}

Note: ${ }^{*} P$ value based on tests comparing IVF and matched NC groups.

TABle 3: Mean and standard deviation (SD) of ages (months) at which motor milestones are attained for IVF/ICSI twins and matched NC twins.

\begin{tabular}{lccccccc}
\hline & \multicolumn{6}{c}{ IVF/ICSI twins } & \multicolumn{5}{c}{ Matched NC twins } & \multirow{2}{*}{$P^{*}$} \\
& $N$ & Mean & $(\mathrm{SD})$ & $N$ & Mean & $(\mathrm{SD})$ & \\
\hline Turning & 2535 & 6.3 & $(1.7)$ & 2473 & 6.4 & $(1.8)$ & 0.870 \\
Sitting & 2553 & 8.7 & $(1.8)$ & 2501 & 8.9 & $(1.8)$ & 0.008 \\
Crawling & 2296 & 9.7 & $(1.7)$ & 2244 & 9.7 & $(1.7)$ & 0.448 \\
Standing alone & 2533 & 12.2 & $(2.4)$ & 2470 & 12.5 & $(2.6)$ & 0.003 \\
Walking alone & 2632 & 14.8 & $(2.4)$ & 2605 & 15.0 & $(2.4)$ & 0.002 \\
\hline
\end{tabular}

Note: ${ }^{*} P$ value based on tests comparing IVF and matched NC groups.

the effect sizes were very small $(0.06,0.08$, and 0.09 , resp. for internalizing problems at age 3 as rated by mother, and at age 7 as rated by mother and teacher).

\section{Discussion}

To date, there are limited data on the development of IVF/ ICSI twins. Therefore, the main purpose of this study was to compare the development of IVF twins with that of naturally conceived (NC) twins with respect to motor milestones, growth, and behavioral problems during childhood. To control for possible confounding factors, only DZ twins were
TABLE 4: SDS height and weight for ages 1 to 12 years for IVF/ICSI twins and matched NC twins.

\begin{tabular}{lccccccc}
\hline \multicolumn{9}{c}{ IVF/ICSI twins } & \multicolumn{3}{c}{ Matched NC twins } & \multirow{2}{*}{$P^{*}$} \\
& $N$ & Mean & $($ SD $)$ & $N$ & Mean & $($ SD $)$ & \\
\hline Height & & & & & & & \\
Age 1 & 2571 & -.42 & $(1.0)$ & 2568 & -.51 & $(1.0)$ & 0.018 \\
Age 2 & 2443 & -.25 & $(1.1)$ & 2421 & -.32 & $(1.1)$ & 0.064 \\
Age 3 & 2262 & -.13 & $(1.1)$ & 2194 & -.18 & $(1.0)$ & 0.249 \\
Age 7 & 1584 & -.09 & $(1.1)$ & 1543 & -.03 & $(1.0)$ & 0.237 \\
Age 10 & 857 & -.05 & $(1.0)$ & 757 & .04 & $(1.0)$ & 0.113 \\
Age 12 & 857 & .08 & $(1.1)$ & 798 & .10 & $(1.0)$ & 0.822 \\
\hline Weight & & & & & & & \\
Age 1 & 2614 & -.44 & $(1.0)$ & 2617 & -.45 & $(1.0)$ & 0.649 \\
Age 2 & 2464 & -.28 & $(1.0)$ & 2451 & -.22 & $(1.0)$ & 0.133 \\
Age 3 & 2261 & -.26 & $(1.0)$ & 2188 & -.21 & $(1.0)$ & 0.160 \\
Age 7 & 1595 & -.36 & $(1.1)$ & 1526 & -.29 & $(1.1)$ & 0.151 \\
Age 10 & 848 & -.24 & $(1.0)$ & 765 & -.13 & $(1.0)$ & 0.086 \\
Age 12 & 843 & -.21 & $(1.0)$ & 794 & -.11 & $(1.0)$ & 0.086 \\
\hline
\end{tabular}

Note: ${ }^{*} P$ value based on tests comparing IVF and matched NC groups.

included, and the NC control group was matched on birth cohort, maternal age and educational level, smoking behavior during pregnancy, and gestational age. Although the perinatal outcomes appear to be slightly worse in IVF twins, the course of postnatal development is very similar in IVF and NC twin children.

For the IVF pregnancies, maternal age was higher and more mothers were primiparous compared to NC pregnancies. Both factors increased the risks for adverse effects on perinatal outcomes. In our data, this was reflected in the higher proportion of preterm births in the IVF group compared to the NC twins. To control for these differences in demographic and maternal characteristics, we matched on these variables. After matching, the results suggest a slight effect of IVF/ICSI treatment on birth weight and length and time in incubator. This effect is probably due to parity. When only primiparous mothers were included in the analyses, effects were no longer significant. Our results agree with those of studies that controlled for chorionicity $[11,13]$ but not with a recent meta-analysis that suggested more preterm births and lower birth weight in IVF pregnancies [14]. Results among studies may differ because all the studies included different control variables. In the meta-analysis there was no adjustment for chorionicity, while this factor might be an important factor in the comparison of perinatal outcomes between IVF/ICSI and NC twins [13]. So, it seems that IVF pregnancies have a more adverse outcome, but it is an open question whether this is due to the IVF treatment itself.

In agreement with other studies $[7,52]$, we found that caesarean sections (CSs) occur more frequently in the IVF group. Even after correction for parity, the rate of CSs is higher for IVF births than for NC births. This higher rate may reflect an increase in the deliberate choice for elective CSs in IVF pregnancies. As an IVF birth is often the first birth after a the history of infertility [53], both the physician 
TABle 5: Number, mean and standard deviation (SD) for internalizing problems (int), externalizing problems (ext) and attention problems (AP) at age $3,7,10$, and 12 years by rater (mother, father, teacher) for IVF/ICSI twins and matched NC twins.

\begin{tabular}{|c|c|c|c|c|c|c|c|}
\hline & \multicolumn{3}{|c|}{ IVF/ICSI twins } & \multicolumn{3}{|c|}{ Matched NC twins } & \multirow{2}{*}{$P^{*}$} \\
\hline & $N$ & Mean & $(\mathrm{SD})$ & $N$ & Mean & $(\mathrm{SD})$ & \\
\hline \multicolumn{8}{|l|}{ Age 3} \\
\hline int mother & 2808 & 4.73 & $(4.0)$ & 2777 & 4.48 & (3.9) & 0.040 \\
\hline int father & 2029 & 4.40 & (3.8) & 1999 & 4.28 & (3.7) & 0.408 \\
\hline ext mother & 2799 & 15.86 & $(9.6)$ & 2776 & 15.40 & $(9.7)$ & 0.147 \\
\hline ext father & 2015 & 14.52 & $(9.0)$ & 1992 & 14.50 & $(9.3)$ & 0.893 \\
\hline \multicolumn{8}{|l|}{ Age 7} \\
\hline int mother & 1850 & 4.91 & $(4.8)$ & 1810 & 4.53 & $(4.8)$ & 0.048 \\
\hline int father & 1325 & 3.72 & $(4.0)$ & 1274 & 3.47 & $(4.1)$ & 0.211 \\
\hline int teacher & 1130 & 5.20 & $(5.6)$ & 1110 & 4.69 & $(5.2)$ & 0.039 \\
\hline ext mother & 1879 & 7.11 & $(6.3)$ & 1830 & 7.20 & $(6.8)$ & 0.721 \\
\hline ext father & 1350 & 6.20 & $(5.8)$ & 1288 & 6.32 & $(6.2)$ & 0.686 \\
\hline ext teacher & 1151 & 4.49 & (6.8) & 1130 & 4.51 & $(7.0)$ & 0.969 \\
\hline AP mother & 1888 & 2.95 & (2.9) & 1851 & 2.79 & $(2.9)$ & 0.126 \\
\hline AP father & 1354 & 2.49 & (2.6) & 1291 & 2.42 & $(2.6)$ & 0.548 \\
\hline AP teacher & 1152 & 5.90 & $(6.7)$ & 1131 & 5.51 & $(6.2)$ & 0.209 \\
\hline \multicolumn{8}{|l|}{ Age 10} \\
\hline int mother & 993 & 5.16 & $(5.3)$ & 899 & 4.96 & (5.5) & 0.514 \\
\hline int father & 697 & 3.51 & $(4.1)$ & 617 & 3.72 & $(4.8)$ & 0.487 \\
\hline int teacher & 877 & 5.57 & (5.9) & 821 & 5.05 & $(5.5)$ & 0.101 \\
\hline ext mother & 999 & 6.25 & $(6.0)$ & 909 & 6.52 & $(6.6)$ & 0.427 \\
\hline ext father & 701 & 4.92 & $(5.0)$ & 624 & 5.33 & $(6.1)$ & 0.271 \\
\hline ext teacher & 898 & 4.85 & (7.8) & 834 & 5.13 & $(7.5)$ & 0.461 \\
\hline AP mother & 1005 & 2.98 & (2.9) & 913 & 2.92 & $(3.1)$ & 0.678 \\
\hline AP father & 704 & 2.41 & (2.6) & 626 & 2.53 & $(2.8)$ & 0.490 \\
\hline AP teacher & 900 & 5.81 & $(6.5)$ & 836 & 6.07 & $(6.8)$ & 0.387 \\
\hline \multicolumn{8}{|l|}{ Age 12} \\
\hline int mother & 974 & 4.60 & (5.6) & 966 & 4.52 & $(5.6)$ & 0.759 \\
\hline int father & 690 & 3.57 & $(4.5)$ & 658 & 3.59 & $(4.6)$ & 0.936 \\
\hline int teacher & 392 & 4.80 & (5.5) & 355 & 4.67 & (5.4) & 0.807 \\
\hline ext mother & 979 & 5.35 & $(6.0)$ & 966 & 5.45 & $(6.3)$ & 0.792 \\
\hline ext father & 692 & 4.40 & (5.3) & 661 & 4.59 & $(5.4)$ & 0.617 \\
\hline ext teacher & 400 & 3.91 & (6.5) & 360 & 4.61 & $(8.6)$ & 0.254 \\
\hline AP mother & 988 & 2.60 & (2.9) & 972 & 2.73 & $(3.1)$ & 0.395 \\
\hline AP father & 692 & 2.28 & (2.6) & 669 & 2.34 & $(2.8)$ & 0.749 \\
\hline AP teacher & 402 & 5.37 & $(6.2)$ & 360 & 5.44 & $(6.5)$ & 0.923 \\
\hline
\end{tabular}

Note: ${ }^{*} P$ value based on tests comparing IVF and matched NC groups.

and mother may be more worried about delivery than in NC pregnancies [52] and may more often plan a CS in advance. However, this does not entirely explain the increase of number of CSs in the IVF group. For a number of the deliveries in our study it was known whether the CS was elective or not. Among all CSs, we found that in the matched NC group of women of 35 years or older, $44 \%$ of the CSs were unexpected and $56 \%$ were agreed on beforehand. In the IVF group of older women, $57 \%$ of the CSs were unexpected, and $43 \%$ were elective CSs. The increased rate of CSs in the IVF group is partly due to deliberate choice for elective CS and partly to an increase of emergency CSs, at least in women aged 35 years or older. It cannot be excluded that IVF itself may be a risk factor for the increased rate of CSs.

There are almost no studies that compared growth of IVF and NC twins. Until age 3, earlier studies found no evidence for differences in growth measures between IVF and NC twins $[23,24]$. The present study investigated growth from birth to 12 years of age and found no evidence for differences in height and weight between IVF and matched NC twins. However, weight and height of the twins are lower than their peers in the Dutch population. Estourgie-van Burk et al. [54] described the growth pattern of twins from birth to 18 years of age. At the age of 4, twins were comparable on height with peers of the Dutch population. It seems that the twins in our study caught up at a later age. At age 7 , the twins were still smaller than their peers. This may be explained by the relatively high rate of preterm births in both the IVF twins and matched NC group, as matching was done on gestational age. The same pattern seems to occur for weight. In the study of Estourgie-van Burk et al. twins had a SDS of -0.14 at age 4, while we observed an SDS of -0.30 at age 7 . Weight at age 12 is still behind of that of children in the Dutch population, but height is similar to that of the children in the Dutch population. The different growth pattern for weight and height is also seen in the study of Estourgie-van Burk et al. [54]. The growth pattern of height and weight seems not to be different between IVF and NC control twins.

In agreement with other studies we did not observe a delay of the gross motor development in the IVF twins compared to the NC twins [23, 27-30]. A notable finding was that IVF twins achieved certain motor milestones earlier compared to NC control twins. The vast majority of the IVF twins are the first-born children after a period of involuntary childlessness. Earlier research showed that mothers of IVF twins were more emotionally involved with their child and interacted more with their child than mothers of NC children [20,55]. Probably, parents of a first-born child push their child more to achieve motor milestones than parents of children with older siblings. To verify this in our study, we compared the attainment of motor milestones between children with and without older siblings. We found that children without an older sibling achieved the motor milestones sitting and walking significantly earlier than the children with an older sibling.

We reported no differences in externalizing behavior and attention problems between the IVF and NC children. The results were found for both parental ratings and were further confirmed by the ratings of the teachers. The confirmation by the teacher ratings is important because the teacher is unaware of the means of conception. The results are in agreement with an earlier study with 5-year old twins [36] which showed no differences in externalizing, internalizing, and hyperactive behaviors between IVF and NC twins as rated by their parents and teacher. However, some studies with IVF singletons showed that parents reported less externalizing behaviors in IVF singletons as compared to NC singletons $[31,34]$. A possible reason might be that mothers of IVF singletons were more emotionally involved and that these mothers were more prone to positively evaluate their 
child. At the younger ages, there was a trend for mothers and teachers to report more internalizing problems. More internalizing problems have been reported for IVF singletons at different ages during childhood [32, 34]. Again, this may result from overprotection by the mother, so that the child may show more anxious and withdrawn behaviors. It should be noted that in our study the effect sizes were very small and that the fathers did not report more internalizing problems for IVF children. It can be concluded that the behavioral development of IVF twins is similar to that of NC twins.

A limitation of the current study is that the results rely entirely on self-reports of the mode of conception. A study of Raj and Morley in 2007 [56] suggested that parents of twins showed less willingness to report about the mode of conception if there was no need for it. However, in a mailing sent to more than 20000 mothers of twins, we did not find evidence for less willingness to answer questions on mode of conception [42]. In the same study we found an agreement between maternal report and hospital records of $94 \%$. A second limitation is that for a part of the IVF pregnancies it is not clear whether the pregnancy is achieved via IVF or ICSI. We have treated IVF and ICSI twins as one group, which may bias the results if the developmental outcomes differ between IVF and ICSI children. In a study that compared problem behaviors in 5 to 8 year-old singletons born after ICSI, IVF, and $\mathrm{NC}$, no differences were found between these groups [57]. In the current version of our survey-1 we now make a distinction between IVF and ICSI, and in the future we may investigate whether there are developmental differences between IVF and ICSI children.

In summary, children born after IVF develop similarly to NC children. Regarding growth, motor development, and behavior problems IVF twins do not differ from NC twins. Parents conceiving twins via IVF can assume that the development of their children will be similar to that of NC twins. Nonetheless, the risk of adverse obstetric outcome in IVF twin remains considerably higher than in NC twins and should not be underestimated [58].

\section{Acknowledgments}

The authors thank all twin families who took part. The study was supported by Spinozapremie (NWO/SPI 56-464-14192); European Research Council Genetics of Mental Illness (ERC230374); twin-family database for behavior genetics and genomics studies' (NWO 480-04-004); a collaborative study of the genetics of DZ twinning (NIH R01 HD042157-01A1). Bartels is financially supported by a senior fellowship of the EMGO+ Institute for Health and Care Research.

\section{References}

[1] J. A. M. Kremer, W. Beekhuizen, R. S. G. M. Bots et al., "The results of in vitro fertilisation in the Netherlands, 1996-2000," Nederlands Tijdschrift voor Geneeskunde, vol. 146, no. 49, pp. 2358-2363, 2002.

[2] J. A. M. Kremer, R. S. G. M. Bots, B. Cohlen et al., "Ten years of results of in-vitro fertilisation in the Netherlands 1996-2005,"
Nederlands Tijdschrift voor Geneeskunde, vol. 152, no. 3, pp. 146-152, 2008.

[3] M. C.W. Scholtes, A. P. Schmoutziguer, and G. H. Zeilmaker, "Intracytoplasmic sperm injection, a substantial improvement in the treatment of male infertility," Nederlands Tijdschrift voor Geneeskunde, vol. 139, no. 11, pp. 554-558, 1995.

[4] E. J. De Boer, F. E. van Leeuwen, I. Den Tonkelaar, C. A. M. Jansen, D. D. M. Braat, and C. W. Burger, "Methods and results of in-vitro fertilisation in the Netherlands in the years 19831994," Nederlands Tijdschrift voor Geneeskunde, vol. 148, no. 29, pp. 1448-1455, 2004.

[5] A. K. Ludwig, A. G. Sutcliffe, K. Diedrich, and M. Ludwig, "Post-neonatal health and development of children born after assisted reproduction: a systematic review of controlled studies," European Journal of Obstetrics Gynecology and Reproductive Biology, vol. 127, no. 1, pp. 3-25, 2006.

[6] M. Voigt, N. Rochow, M. Zygmunt, S. Straube, K. T. M. Schneider, and V. Briese, "Risks of pregnancy and birth, birth presentation, and mode of delivery in relation to the age of primiparous women," Zeitschrift fur Geburtshilfe und Neonatologie, vol. 212, no. 6, pp. 206-210, 2008.

[7] S. V. Glinianaia, M. A. Obeysekera, S. Sturgiss, and R. Bell, "Stillbirth and neonatal mortality in monochorionic and dichorionic twins: a population-based study," Human Reproduction, vol. 26, no. 9, pp. 2549-2557, 2011.

[8] H. Verstraelen, S. Goetgeluk, C. Derom et al., "Preterm birth in twins after subfertility treatment: population based cohort study," British Medical Journal, vol. 331, no. 7526, pp. 11731176, 2005.

[9] C. B. Lambalk and M. van Hooff, "Natural versus induced twinning and pregnancy outcome: a Dutch nationwide survey of primiparous dizygotic twin deliveries," Fertility and Sterility, vol. 75, no. 4, pp. 731-736, 2001.

[10] B. Källén, O. Finnström, A. Lindam, E. Nilsson, K. G. Nygren, and P. O. Olausson, "Selected neonatal outcomes in dizygotic twins after IVF versus non-IVF pregnancies," BJOG, vol. 117, no. 6, pp. 676-682, 2010.

[11] J. Joy, N. McClure, and I. E. Cooke, "A comparison of spontaneously conceived twins and twins conceived by artificial reproductive technologies," Journal of Obstetrics and Gynaecology, vol. 28, no. 6, pp. 580-585, 2008.

[12] E. Vasario, V. Borgarello, C. Bossotti et al., "IVF twins have similar obstetric and neonatal outcome as spontaneously conceived twins: a prospective follow-up study," Reproductive BioMedicine Online, vol. 21, no. 3, pp. 422-428, 2010.

[13] H. Yang, Y. S. Choi, K. H. Nam, J. Y. Kwon, Y. W. Park, and Y. H. Kim, "Obstetric and perinatal outcomes of dichorionic twin pregnancies according to methods of conception: spontaneous versus in-vitro fertilization," Twin Research and Human Genetics, vol. 14, no. 1, pp. 98-103, 2011.

[14] S. D. McDonald, Z. Han, S. Mulla, A. Ohlsson, J. Beyene, and K. E. Murphy, "Preterm birth and low birth weight among in vitro fertilization twins: a systematic review and metaanalyses," European Journal of Obstetrics Gynecology and Reproductive Biology, vol. 148, no. 2, pp. 105-113, 2010.

[15] K. J. Middelburg, M. J. Heineman, A. F. Bos, and M. HaddersAlgra, "Neuromotor, cognitive, language and behavioural outcome in children born following IVF or ICSI-a systematic review," Human Reproduction Update, vol. 14, no. 3, pp. 219231, 2008.

[16] K. Wagenaar, J. Huisman, P. T. Cohen-Kettenis, and H. Adelemarre-van De Waal, "An overview of studies on early development, cognition, and psychosocial well-being in children 
born after in vitro fertilization," Journal of Developmental and Behavioral Pediatrics, vol. 29, no. 3, pp. 219-230, 2008.

[17] H. Colpin and S. Soenen, "Parenting and psychosocial development of IVF children: a follow-up study," Human Reproduction, vol. 17, no. 4, pp. 1116-1123, 2002.

[18] H. Colpin, A. De Munter, K. Nys, and L. Vandemeulebroecke, "Parenting stress and psychosocial well-being among parents with twins conceived naturally or by reproductive technology," Human Reproduction, vol. 14, no. 12, pp. 3133-3137, 1999.

[19] F. Olivennes, S. Golombok, C. Ramogida, and J. Rust, "Behavioral and cognitive development as well as family functioning of twins conceived by assisted reproduction: findings from a large population study," Fertility and Sterility, vol. 84, no. 3, pp. 725-733, 2005.

[20] F. van Balen, "Child-rearing following in vitro fertilization," Journal of Child Psychology and Psychiatry and Allied Disciplines, vol. 37, no. 6, pp. 687-693, 1996.

[21] M. Ceelen, M. M. van Weissenbruch, J. P. W. Vermeiden, F. E. van Leeuwen, and H. A. Delemarre-van de Waal, "Growth and development of children born after in vitro fertilization," Fertility and Sterility, vol. 90, no. 5, pp. 1662-1673, 2008.

[22] M. Ceelen, M. M. van Weissenbruch, J. Prein et al., "Growth during infancy and early childhood in relation to blood pressure and body fat measures at age 8-18 years of IVF children and spontaneously conceived controls born to subfertile parents," Human Reproduction, vol. 24, no. 11, pp. 2788-2795, 2009.

[23] S. Koivurova, A. L. Hartikainen, U. Sovio, M. Gissler, E. Hemminki, and M. R. Järvelin, "Growth, psychomotor development and morbidity up to 3 years of age in children born after IVF," Human Reproduction, vol. 18, no. 11, pp. 23282336, 2003.

[24] J. M. Brandes, A. Scher, J. Itzkovits, I. Thaler, M. Sarid, and R. Gershoni-Baruch, "Growth and development of children conceived by in vitro fertilization," Pediatrics, vol. 90, no. 3, pp. 424-429, 1992.

[25] M. Ceelen, M. M. van Weissenbruch, J. C. Roos, J. P. W. Vermeiden, F. E. van Leeuwen, and H. A. Delemarre-van De Waal, "Body composition in children and adolescents born after in vitro fertilization or spontaneous conception," Journal of Clinical Endocrinology and Metabolism, vol. 92, no. 9, pp. 3417-3423, 2007.

[26] M. Knoester, F. M. Helmerhorst, J. P. Vandenbroucke, L. A. J. van der Westerlaken, F. J. Walther, and S. Veen, "Perinatal outcome, health, growth, and medical care utilization of 5to 8-year-old intracytoplasmic sperm injection singletons," Fertility and Sterility, vol. 89, no. 5, pp. 1133-1146, 2008.

[27] I. Ponjaert-Kristoffersen, M. Bonduelle, J. Barnes et al., "International collaborative study of intracytoplasmic sperm injection-conceived, in vitro fertilization-conceived, and naturally conceived 5-year-old child outcomes: cognitive and motor assessments," Pediatrics, vol. 115, no. 3, pp. e283-e289, 2005.

[28] A. Ludwig, A. Katalinic, U. Thyen, A. G. Sutcliffe, K. Diedrich, and M. Ludwig, "Neuromotor development and mental health at 5.5 years of age of singletons born at term after intracytoplasmatic sperm injection ICSI: results of a prospective controlled single-blinded study in Germany," Fertility and Sterility, vol. 91, no. 1, pp. 125-132, 2009.

[29] L. Leunens, S. Celestin-Westreich, M. Bonduelle, I. Liebaers, and I. Ponjaert-Kristoffersen, "Cognitive and motor development of 8-year-old children born after ICSI compared to spontaneously conceived children," Human Reproduction, vol. 21, no. 11, pp. 2922-2929, 2006.
[30] M. Knoester, J. P. Vandenbroucke, F. M. Helmerhorst, L. A. J. van der Westerlaken, F. J. Walther, and S. Veen, "Matched follow-up study of 5-8 year old ICSI-singletons: comparison of their neuromotor development to IVF and naturally conceived singletons," Human Reproduction, vol. 22, no. 6, pp. 1638-1646, 2007.

[31] J. Barnes, A. G. Sutcliffe, I. Kristoffersen et al., "The influence of assisted reproduction on family functioning and children's socio-emotional development: results from a European study," Human Reproduction, vol. 19, no. 6, pp. 1480-1487, 2004.

[32] M. Jongbloed-Pereboom, K. J. Middelburg, M. J. Heineman, A. F. Bos, and M. Hadders-Algra, "The Groningen assisted reproductive technologies cohort study: developmental status and behavior at 2 years," Fertility and Sterility, vol. 95, no. 7, pp. 2283-2289, 2011.

[33] M. Knoester, F. M. Helmerhorst, L. A. J. van der Westerlaken, F. J. Walther, and S. Veen, "Matched follow-up study of 5-8-yearold ICSI singletons: child behaviour, parenting stress and child (health-related) quality of life," Human Reproduction, vol. 22, no. 12, pp. 3098-3107, 2007.

[34] K. Wagenaar, M. M. van Weissenbruch, D. L. Knol, P. T. Cohen-Kettenis, H. A. Delemarre-van de Waal, and J. Huisman, "Behavior and socioemotional functioning in 9-18-yearold children born after in vitro fertilization," Fertility and Sterility, vol. 92, no. 6, pp. 1907-1914, 2009.

[35] K. Wagenaar, M. M. van Weissenbruch, F. E. van Leeuwen et al., "Self-reported behavioral and socioemotional functioning of 11- to 18-year-old adolescents conceived by in vitro fertilization," Fertility and Sterility, vol. 95, no. 2, pp. 611-616, 2011.

[36] L. A. Tully, T. E. Moffitt, and A. Caspi, "Maternal adjustment, parenting and child behaviour in families of school-aged twins conceived after IVF and ovulation induction," Journal of Child Psychology and Psychiatry and Allied Disciplines, vol. 44, no. 3, pp. 316-325, 2003.

[37] M. Bartels, C. E. M. van Beijsterveldt, E. M. Derks et al., "Young Netherlands Twin Register (Y-NTR): a longitudinal multiple informant study of problem behavior," Twin Research and Human Genetics, vol. 10, no. 1, pp. 3-11, 2007.

[38] D. I. Boomsma, E. J. C. De Geus, J. M. Vink et al., "Netherlands Twin Register: from twins to twin families," Twin Research and Human Genetics, vol. 9, no. 6, pp. 849-857, 2006.

[39] D. I. Boomsma, J. M. Vink, T. C. E. M. van Beijsterveldt et al., "Netherlands Twin Register: a focus on longitudinal research," Twin Research, vol. 5, no. 5, pp. 401-406, 2002.

[40] M. J. H. Rietveld, J. C. van der Valk, I. L. Bongers, T. M. Stroet, P. E. Slagboom, and D. I. Boomsma, "Zygosity diagnosis in young twins by parental report," Twin Research, vol. 3, no. 3, pp. 134-141, 2000.

[41] C. Hoekstra, G. Willemsen, T. C. E. M. van Beijsterveldt, G. W. Montgomery, and D. I. Boomsma, "Familial twinning and fertility in Dutch mothers of twins," American Journal of Medical Genetics A, vol. 146, no. 24, pp. 3147-3156, 2008.

[42] C. E. M. T. van Beijsterveldt, C. Hoekstra, R. Schats, G. W. Montgomery, G. Willemsen, and D. I. Boomsma, "Mode of conception of twin pregnancies: willingness to reply to survey items and comparison of survey data to hospital records," Twin Research and Human Genetics, vol. 11, no. 3, pp. 349-351, 2008.

[43] J. M. Langendonk, C. E. M. van Beijsterveldt, S. I. Brouwer, T. Stroet, J. J. Hudziak, and D. I. Boomsma, "Assessment of motor milestones in twins," Twin Research and Human Genetics, vol. 10, no. 6, pp. 835-839, 2007. 
[44] S. I. Brouwer, T. C. E. M. van Beijsterveldt, M. Bartels, J. J. Hudziak, and D. I. Boomsma, "Influences on achieving motor milestones: a twin-singleton study," Twin Research and Human Genetics, vol. 9, no. 3, pp. 424-430, 2006.

[45] T. M. Achenbach, Manual for the Child Behavior Checklist/23 and 1992 Profile, University of Vermont, Department of Psychiatry, Burlington, Vt, USA, 1992.

[46] H. M. Koot, E. J. C. G. van Den Oord, F. C. Verhulst, and D. I. Boomsma, "Behavioral and emotional problems in young preschoolers: cross-cultural testing of the validity of the child behavior checklist/2-3," Journal of Abnormal Child Psychology, vol. 25, no. 3, pp. 183-196, 1997.

[47] T. M. Achenbach, Manual for the Child Behavior Checklist/418 and 1991 Profile, University of Vermont, Department of Psychiatry, Burlington, Vt, USA, 1991.

[48] T. M. Achenbach, Manual for the Teacher's Report Form and 1911 Profile, University of Vermont, Department of Psychiatry, Burlington, Vt, USA, 1996.

[49] A. M. Fredriks, S. van Buuren, J. M. Wit, and S. P. VerlooveVanhorick, "Body index measurements in 1996-7 compared with 1980," Archives of Disease in Childhood, vol. 82, no. 2, pp. 107-112, 2000.

[50] A. M. Fredriks, S. van Buuren, R. J. F. Burgmeijer et al., "Continuing positive secular growth change in the Netherlands 1955-1997," Pediatric Research, vol. 47, no. 3, pp. 316-323, 2000.

[51] SPSS, SPSS Advanced Models 12.0, SPSS, Chicago, Ill, USA, 2003.

[52] O. Shebl, T. Ebner, A. Sir, M. Sommergruber, and G. Tews, "The role of mode of conception in the outcome of twin pregnancies," Minerva Ginecologica, vol. 61, no. 2, pp. 141$152,2009$.

[53] C. A. McMahon, J. A. Ungerer, J. Beaurepaire, C. Tennant, and D. Saunders, "Anxiety during pregnancy and fetal attachment after in-vitro fertilization conception," Human Reproduction, vol. 12, no. 1, pp. 176-182, 1997.

[54] G. F. Estourgie-van Burk, M. Bartels, D. I. Boomsma, and H. A. Delemarre-van de Waal, "Body size of twins compared with siblings and the general population: from birth to late adolescence," Journal of Pediatrics, vol. 156, no. 4, pp. 586-591, 2010.

[55] S. Golombok, A. Brewaeys, R. Cook et al., "The European study of assisted reproduction families: family functioning and child development," Human Reproduction, vol. 11, no. 10, pp. 2324-2331, 1996.

[56] S. Raj and R. Morley, "'Are you asking me if we had sex to conceive?" To whom do parents of twins disclose mode of conception and what do they feel about being asked?" Twin Research and Human Genetics, vol. 10, no. 6, pp. 886-891, 2007.

[57] M. Knoester, F. M. Helmerhorst, J. P. Vandenbroucke, L. A. J. van der Westerlaken, F. J. Walther, and S. Veen, "Cognitive development of singletons born after intracytoplasmic sperm injection compared with in vitro fertilization and natural conception," Fertility and Sterility, vol. 90, no. 2, pp. 289-296, 2008.

[58] A. Pinborg, A. Loft, and A. N. Andersen, "Neonatal outcome in a Danish national cohort of 8602 children born after in vitro fertilization or intracytoplasmic sperm injection: the role of twin pregnancy," Acta Obstetricia et Gynecologica Scandinavica, vol. 83, no. 11, pp. 1071-1078, 2004. 


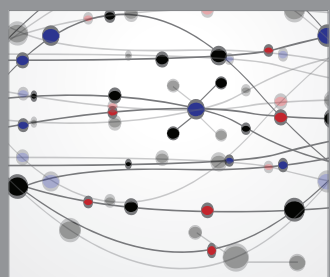

The Scientific World Journal
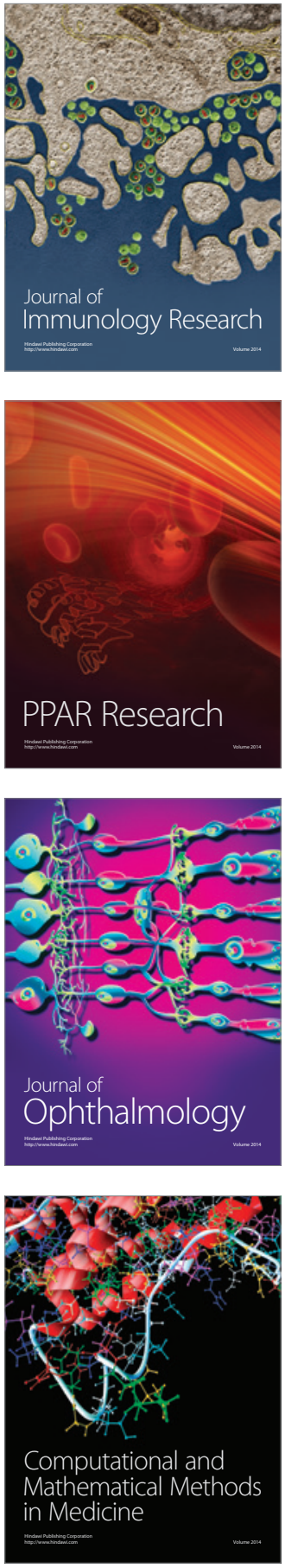

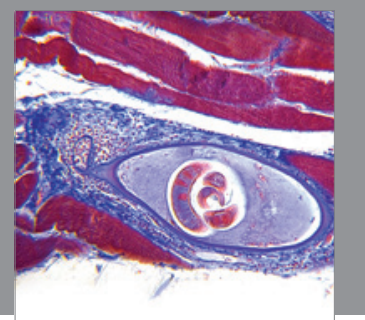

Gastroenterology

Research and Practice
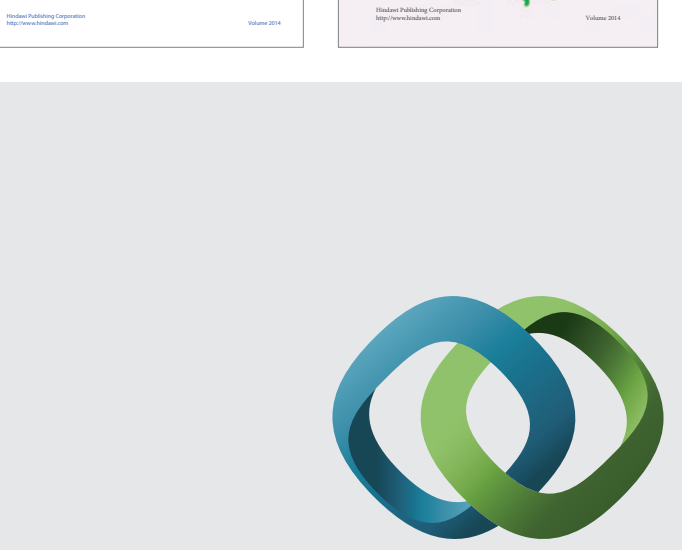

\section{Hindawi}

Submit your manuscripts at

http://www.hindawi.com
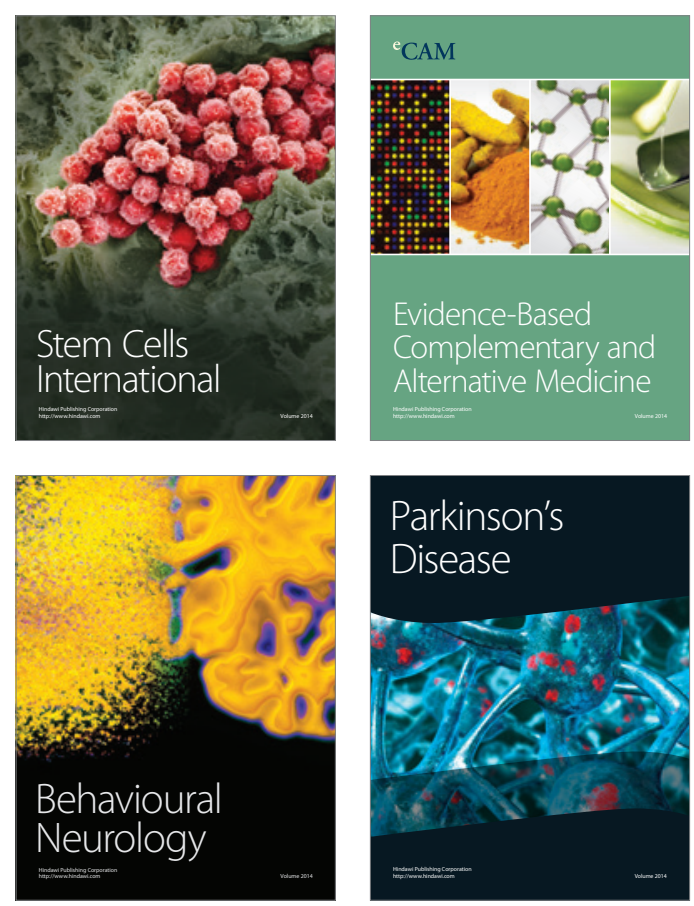

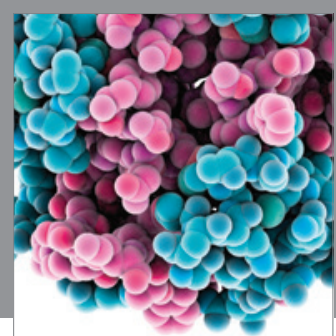

Journal of
Diabetes Research

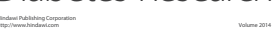

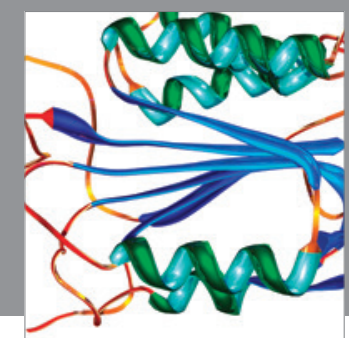

Disease Markers
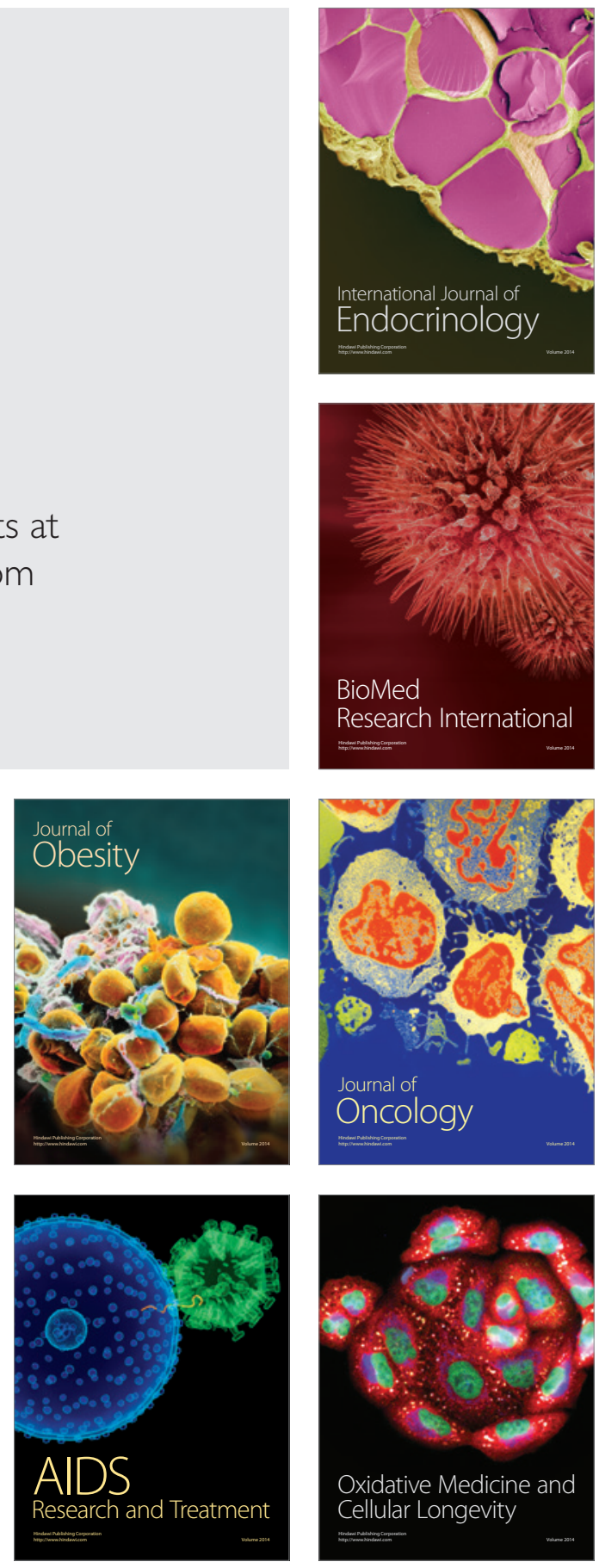